\title{
Intrathoracic jugular vein avulsion after blunt chest trauma
}

\author{
Ernesto Hayn, MD, Leonard O. Barrett, MD, J. Christopher DiGiacomo, MD, and Gerald W. Shaftan, MD, East Meadow, NY
}

I njuries to the vascular structures of the thoracic outlet are infrequent and are typically accompanied by massive hemorrhage. As a result of improvements in the emergency medical system and rapid prehospital transport, however, some of these patients are now arriving at the trauma center before they exsanguinate from the injury. Most injuries of the cervicothoracic area are combined arterial and venous injuries and are produced mainly by penetrating trauma. ${ }^{1,2}$ Isolated venous injury of this region after blunt chest trauma is exceptionally rare. ${ }^{2}$

\section{Clinical Summary}

A 60-year-old man with no significant medical history was caught between 2 trucks and sustained a crushing injury to his chest. In the trauma resuscitation area he was found to have moderate respiratory distress and a small right supraclavicular hematoma without a bruit or thrill. There was no facial edema, cyanosis, jugular venous distention, plethora, petechiae, or retinal-conjunctival or ecchymotic hemorrhages of the face or upper part of the chest. Peripheral pulses were intact.

Chest radiography demonstrated fractures of the bony chest wall and right-sided pneumothorax (Figure 1). His respiratory status improved after placement of a right-side tube thoracostomy, and the patient underwent evaluation by means of computed tomography (CT).

CT of the chest revealed a right apical supraclavicular hematoma, fracture at the medial one third of the right clavicle, multiple bilateral rib fractures, an occult left pneumothorax, and a retropleural hematoma near the apex of the left lung (Figure 2). A left-sided chest tube was placed, and the patient promptly underwent angiographic evaluation, which showed no evidence of arterial extravasation of contrast material during the arterial phase (Figure 3). The venous phase displayed extravasation at the confluence of the right internal jugular vein with the innominate vein (Figure 4). At this time, the patient remained metastable, with a falling hematocrit level and continued intravenous fluid needs to

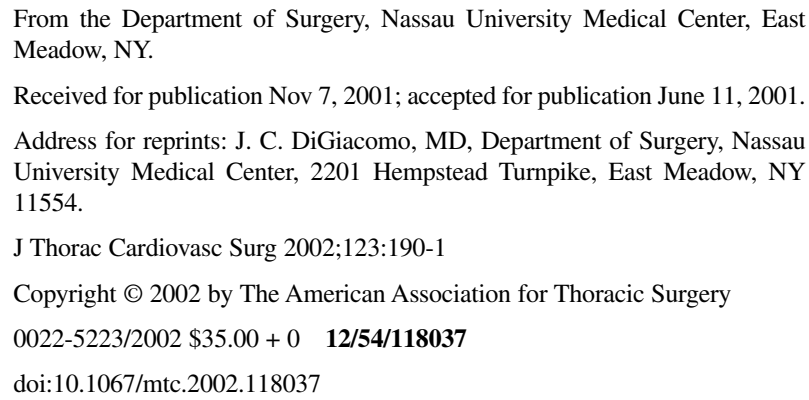

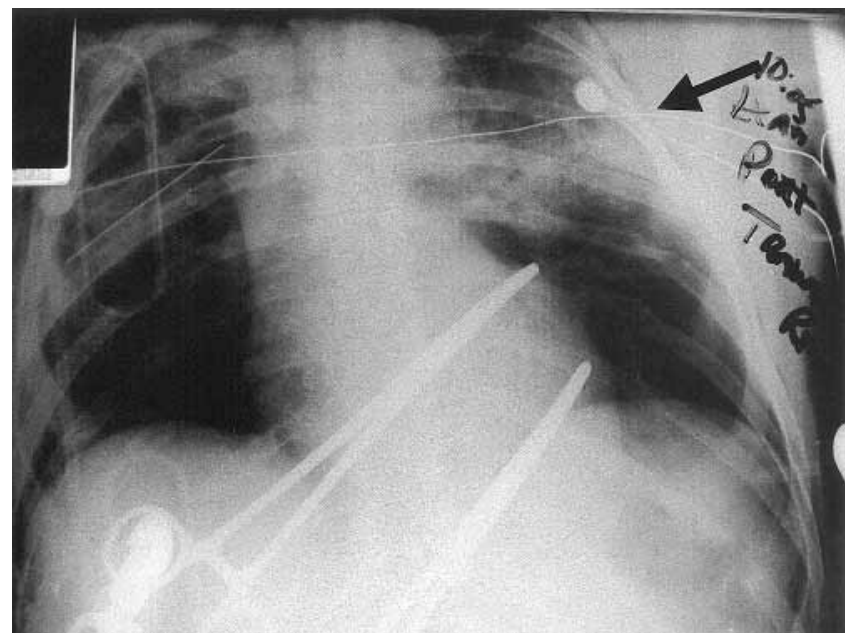

Figure 1. Chest radiograph from the trauma room demonstrating rib fractures (arrow). The right chest tube is in place.

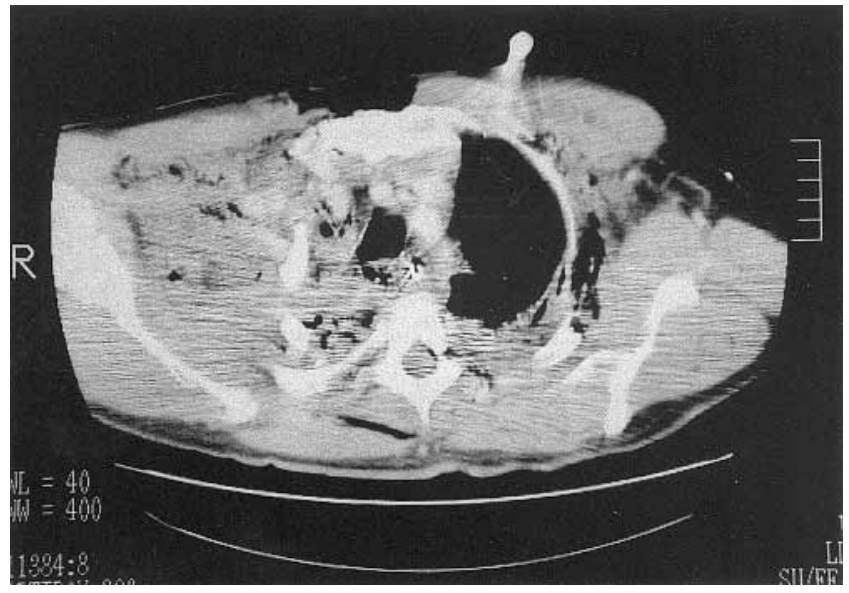

Figure 2. CT scan of the chest demonstrating the right apical supraclavicular hematoma and right clavicle fracture and a retropleural hematoma in the upper left side of the chest. The multiple bilateral rib fractures and occult left pneumothorax are not demonstrated on this selected cut.

maintain hemodynamic stability. He was taken to the operating room for repair.

\section{Operation}

The chest was opened through a median sternotomy with extension to the right side of the neck. Once the avulsion of the right internal jugular vein at the junction of the innominate vein was identified, 


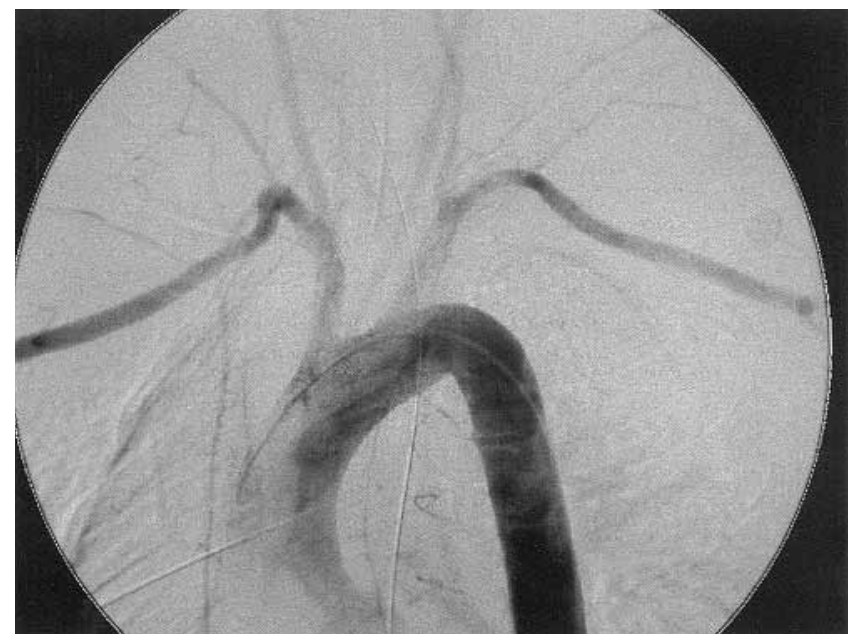

Figure 3. Thoracic arteriogram demonstrating no injuries.

the right internal jugular vein was ligated, and the innominate vein defect was closed with a running 4-0 polypropylene suture without difficulty.

\section{Discussion}

Thoracic vessel injuries may be associated with blunt thoracic trauma. ${ }^{3}$ Although blunt thoracic trauma accounts for the majority of injuries to the proximal innominate artery and the descending thoracic aorta, blunt venous injury is rare. ${ }^{2,3}$ In fact, Fisher and colleagues $^{3}$ reported only one pulmonary vein rupture among 161 cases of blunt thoracic trauma. Graham and coworkers ${ }^{4}$ found a single innominate vein injury among 42 cases of innominate vascular injuries over a 35-year period. Recently, Bowles and colleagues $^{5}$ reviewed the world literature and found 11 cases of azygos vein rupture from blunt trauma, all caused by abrupt and marked deceleration.

The patient presented above is unique not only because of the rarity of his injury but also because of the mechanism through which he was injured. The blunt-compression injury produced no signs or symptoms consistent with a sudden increase in intravascular venous pressure, and the isolated venous injury occurred without an associated arterial injury. The only signs of thoracic trauma were the multiple rib fractures.

Angiography remains the gold standard for the diagnosis of stable patients with thoracic vascular injury. ${ }^{6}$ Angiography provides the detailed anatomic information necessary for planning the operative approach, and venous injuries can be identified through the venous phase. Although the resolution of the helical CT scan is remarkable, it has not been demonstrated to be reliable in ruling out vascular injury in the chest or consistently diagnosing thoracic vascular injury with precision, the occasional and sporadic anecdotal reports notwithstanding. 5,7

The approach adopted for the diagnosis and treatment should be based on the clinical condition of the patient. Immediate surgical exploration is warranted for obvious signs of vascular trauma,

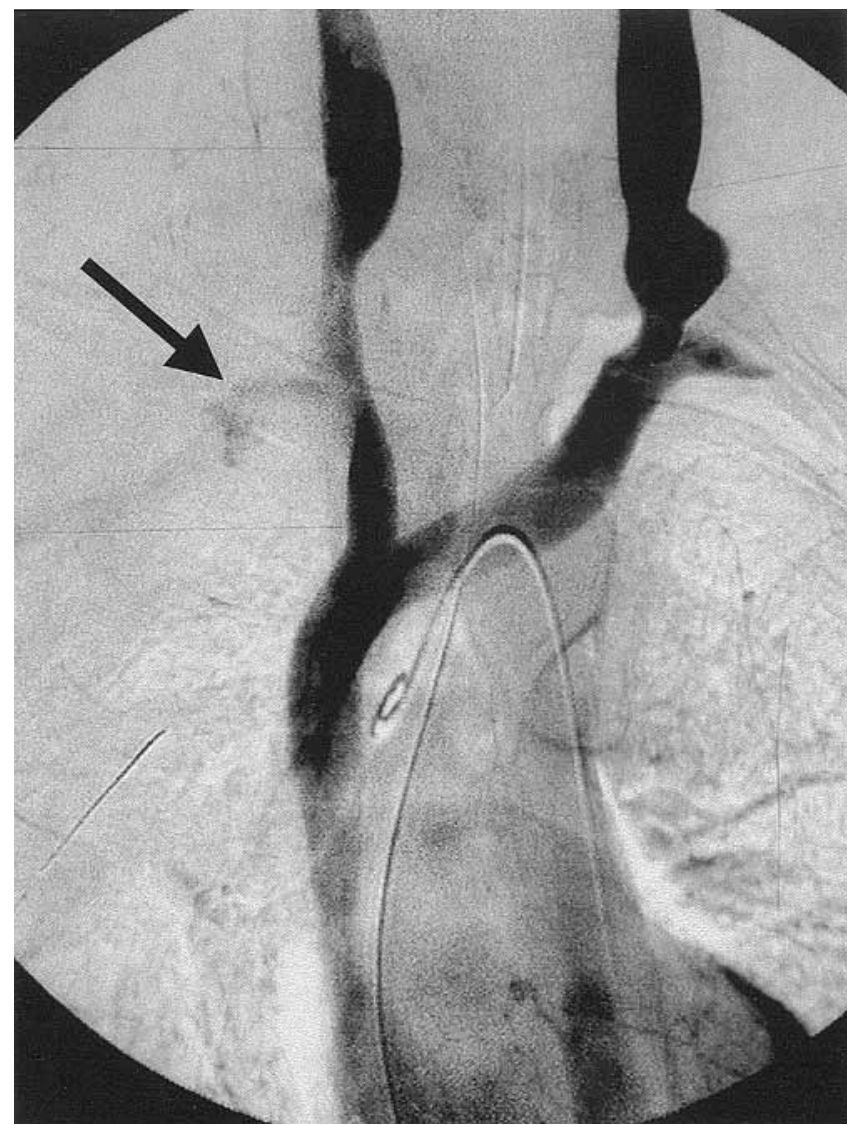

Figure 4. Venous phase of the thoracic arteriogram, demonstrating extravasation at the confluence of the right internal jugular vein and the innominate vein (arrow).

such as active bleeding, expanding hematoma, and hemodynamic instability. In the absence of such signs, however, we continue to advocate diagnosis through angiography and venography.

\section{References}

1. Bricker DL, Noon GP, Beall AC, DeBakey ME. Vascular injuries of the thoracic outlet. J Trauma. 1970;10:1-14.

2. Mattox K. Approaches to trauma involving the major vessels of the thorax. Surg Clin North Am. 1989;69:77-91.

3. Fisher RG, Sanchez-Torres M, Thomas JW, Whigham CJ. Subtle or atypical injuries of the thoracic aorta and brachiocephalic vessels in blunt thoracic trauma. Radiographics. 1997;17:835-49.

4. Graham JM, Feliciano DV, Mattox KL, Beall AC. Innominate vascular injury. J Trauma. 1982;22:647-55.

5. Bowles BJ, Teruya T, Belzberg H, Rivkind AI. Blunt traumatic azygous vein injury diagnosed by computed tomography. J Trauma. 2000;49:776-9.

6. Mattox KL. The use of enhanced computerized CT scanning to diagnose injury to the thoracic aorta. J Trauma. 1999;46:742-5.

7. Patel NH, Stephens KE, Mirvis SE, Shanmuganathan K, Mann FA. Imaging of acute thoracic aortic injury due to blunt trauma. Radiology. 1998;209:335-48 